\title{
Proceedings of the 2020 ISNR Annual Conference (Virtual): Keynote and Plenary Presentations
}

\author{
Selected Abstracts of Conference Keynote and Plenary Presentations at the 2020 International Society \\ for Neurofeedback and Research (ISNR) 28th Annual Conference, Miami, Florida, USA
}

Citation: International Society for Neurofeedback and Research. (2020). Proceedings of the 2020 ISNR Annual Conference (Virtual): Keynote and Plenary Sessions. NeuroRegulation, 7(4), 158-172. https://doi.org/10.15540/nr.7.4.158

Copyright: $\odot$ 2020. ISNR. This is an Open Access article distributed under the terms of the Creative Commons Attribution License (CC-BY).

\section{KEYNOTE PRESENTATIONS}

\section{Neuro and Biofeedback in Neurological \\ Rehabilitation: An Integrative Approach \\ Leon Morales-Quezada \\ Spaulding Neuromodulation Center, HMS, Boston, Massachusetts, USA}

Neurological rehabilitation can improve function, reduce symptoms, and improve the well-being of the patient suffering from diseases, injury, or disorders of the nervous system. A variety of techniques or methods are used for neurological rehabilitation, from physical and speech therapies to advanced robotbased treatments. Applied psychophysiology offers an alternative for patients to improve their neurological deficits; neuro and biofeedback can be applied in the clinical settings to facilitate cognitive and motor functions, or to be used as adjunct intervention for symptoms and conditions associated to neuropathology such as seizures, depression, or autonomic dysfunction.

In his talk, Dr. Morales-Quezada will present the principles of neuroplasticity behind neurological rehabilitation, and how to implement neuro/biofeedback interventions in a third-level rehabilitation hospital. Functional diagnostics and multidisciplinary management will also be covered in this talk.

\section{Neuromodulation Techniques for Altering Brain Plasticity and Cognition \\ William Jamie Tyler \\ Arizona State University, Tempe, Arizona, USA}

Noninvasive neuromodulation systems and devices are used every day around the world for research, to treat medical indications, to enhance human performance, and in other consumer wellness and electronics applications. Our scientific, technical, and engineering efforts have led to the development and deployment of several distinct methods, which will be discussed. Transcranial focused ultrasound (tFUS) provides the highest spatial resolution of all the noninvasive neuromodulation methods. It also enables the focal, noninvasive neuromodulation of deep-brain circuits in humans. Translational efforts have shown promise that tFUS can provide therapeutic benefits for several mental health conditions. We will discuss recent evidence and emerging indications for the use of tFUS in modulating brain plasticity, sensory awareness, decision-making, and mood. Other bottom-up methods targeting brainstem nuclei of the reticular activating system will be discussed. These transdermal electrical nerve stimulation methods include trigeminal nerve stimulation and transdermal auricular vagus nerve stimulation (taVNS), which have been shown to modulate sympathetic tone and enhance brain plasticity by affecting the activity of the locus coeruleus and norepinephrine signaling. We will describe collective efforts using trigeminal nerve stimulation and taVNS to provide active control of sympathetic nervous system activity for regulating alertness, attention, stress, anxiety, learning, and sleep/wake cycles. In all cases we will discuss the central role of quantitative psychophysiological and neurophysiological biomarkers in the iterative development, validation, and verification of these noninvasive neuromodulation methods. Emerging machine learning and artificial intelligence methods now promise to mine large data sets from similarly connected biosensors in real-world environments. Therefore, we will discuss how these approaches are being integrated with noninvasive neuromodulation methods in open-loop and closed-loop manners to develop personalized approaches, advance our understanding of brain and behavior, and enable the optimization of human health and performance. Finally, we will discuss modern regulatory, ethical, and legal considerations for the investigation, use, and commercialization of noninvasive 
neurotechnologies intended to modulate or measure human brain activity in various applications.

\section{Neuromodulation Techniques for Altering Brain Plasticity and Cognition \\ Marty Teicher \\ McLean Hospital, Belmont, Massachusetts, USA}

Exposure to childhood adversity markedly increases the risk of developing mood, anxiety, personality, substance abuse, and psychotic disorders. Recent studies suggest that clinical sequelae may stem, at least in part, from enduring adverse effects on brain development. Generally, early onset and longer duration of abuse have been associated with greater brain changes, but this is an oversimplification. It appears that stress-susceptible brain regions have their own unique sensitive periods (or windows of vulnerability) to the effects of early stress. Further, evidence also suggests that maltreated and nonmaltreated individuals with the same DSM diagnoses are clinically, neurobiologically, and genetically distinct. We refer to the disorder in the maltreated cohort as an ecophenotype and show that it is associated with earlier age of onset, more severe course, more comorbid diagnoses, and poorer response to first-line treatments. A key challenge has been to understand why some individuals appear to be resilient to the psychiatric sequelae of abuse, particularly as they show the same basic array of alterations in stress-susceptible brain regions as comparably maltreated individuals with severe psychopathology. Our recent studies of brain network architecture have identified additional alterations in the brains of maltreated individuals that enable them to effectively compensate and to obtain high levels of mental health. This leads to a new understanding of how recovery occurs and how effective treatments may work-which is not to reverse the effects of maltreatment, but to foster compensatory resilience by altering the connectivity of specific brain regions.

\section{Stress and Stress-Activated Viruses in Public Health, Mental Health and Anticipatory \\ Pathology \\ Roulett Smith \\ Humanized Technologies, Palo Alto, California, USA}

Processes of evolution are among the most awesome developments during the 4.5+ billion years associated with the existence of this Earth. Among the many forces contributing to evolution are climate, tectonic plate movements, and stresses on evolved group behaviors (including common sense). This presentation will focus on the potential public health and mental health consequences of the extraordinary stresses associated with two rare and intertangled contemporaneous events requiring common sense; to wit, the COVID-19 pandemic and outbursts of rage, social unrest, and mass demonstrations associated with George Floyd's death.

For living systems (whether in plants, animals, or other living systems) "fight or flight" mechanisms (and their underlying behaviors and responses to stress) are among the obvious contributors to evolutionary developments. In animals, the autonomic nervous system consists of sympathetic and parasympathetic components. Fight and/or flight mechanisms are associated with the sympathetic nervous system and its associated increases or decreases in various stress hormones (e.g., cortisol/glucocorticoids, catecholamines, thyroid, growth hormone, prolactin, vasopressin, gonadotropins, insulin, etc.). The parasympathetic nervous system regulates relaxation and slows high-energy functions.

Stress also is associated with structural changes. For example, in brain chronic stress can lead to both neurogenesis and cell death in the hippocampus, increases in myelin-producing cells, reduction in gray matter (responsible for high-order cognitive functions), brain shrinkage, losses in short-term and spatial memories, and increased risks for mental illness.

Most reports on the COVID-19 pandemic focus on atrisk subpopulations. These include travelers on ocean liners or military/naval vessels; homeless persons; essential employees; senior citizens (in senior housing, hospice, or nursing facilities); persons in prisons and detention centers; persons with intellectual disabilities; and persons with various comorbidities. It now is anticipated that the COVID19 pandemic will give rise to more than 13 long-term effects on mental health (e.g., depression, posttraumatic stress disorder [PTSD], anxiety disorders, shame, avoidance disorders, burnout, alcoholism and substance abuse, isolation and estrangement, loss of identity or purpose, suicide, etc.). Common sense issues also may affect public and mental health.

During this pandemic, a cohort of youngsters is especially noteworthy for outbreaks of Kawasaki-like autoimmune presentations. This finding suggests that childhood stresses (whether directly or indirectly involving the COVID-19 virus) may be triggering a stress-activated virus known to be associated with 
Kawasaki disease. This further suggests that separate stress-activated viruses also may be significant and evolutionary cofactors in the COVID19 pandemic. Indeed, my earlier research from the 1980s reveals that small RNA particles (snRNPs) encoded by stress-activated gamma herpes viruses (e.g., the Epstein-Barr virus, as well as selected other stress-activated viruses) may be transmissible and infectious (i.e., autovirulence). Autovirulence can give rise to transcription and/or translation errors for host genes (GERRs) and a plethora of downstream disorders.

To date, GERRs are responsible for virtually all autoimmune disorders, many congenital disorders, autism spectrum disorders, many trinucleotide repeat (TNR) disorders, and male homosexuality. Hence, one now can anticipate that the extraordinary stresses associated with the COVID-19 pandemic and concurrent mass demonstrations may give rise to novel future pathologies and other evolutionary outcomes.

In summary, social, physical, environmental, political, and molecular biological factors associated with the COVID-19 pandemic and mass demonstrations may be generators of genetic diversity ... both in the COVID-19 virus and in evolution. We also explore the implications of these consequences ... including common sense.

\section{Recent Psychophysiological Advances of "Spontaneous" Reading Giuseppe Augusto Chiarenza}

Centro Internazionale dei Disturbi di Apprendimento Attenzione Iperattività (CIDAAI), Milano, Italy

The study of goal-directed behavior in normal and dyslexic subjects during spontaneous reading was the guiding thread of my research in the field of neuroevolutionary disorders. Reading can be defined as an active perceptual process of gathering information from the external world that involves attention, memory, articulation, planning, and intention. In this sense, reading can be considered a behavior teleologically purposive. Unfortunately, most of the experimental reading conditions have been restricted to automatic presentation of stimuli on a computer screen where the subjects had to passively look or read silently. Furthermore, there are no studies describing the modifications of the brain electrical activity when the subjects in a self-paced condition read single letters aloud. This meant that the components related to planning and intention were not examined. For this purpose, the chronology of movement-related potentials during skilled actions will be presented which shows that dyslexia is not only a defect of the auditory and/or visual perceptual processes but may also be considered as a praxic disorder in which praxic abilities, such as motor programming, sequential and sensory-motor integration, and error system analysis show reduced efficiency. Furthermore, when comparing the reading-related potentials (RRPs) during passive and self-paced condition, significant differences emerge regarding the time course and spatial distribution on the scalp of these potentials. Self-paced reading aloud is characterized by the most numerous and significant modifications of RRPs morphology in all the cerebral areas. The self-paced condition and the acquisition of extra cerebral signals, as subjects' voice, EMG activity of forearm and of lips, allowed to identify the potentials occurring before, during, and after reading and the cerebral areas mostly involved in each of these periods. The potentials belonging to the prelexical period are undoubtedly related to perceptual analysis of stimuli, while those occurring during the lexical and postlexical periods likely represent reafferent activity for the control of linguistic processes.

Important and significant differences have also been described when comparing different clinical subtypes of dyslexia. This was possible thanks to the direct self-paced reading and spelling test developed by Chiarenza (2010), inspired by the reading and writing model proposed by Boder (1973). Boder described three main subtypes of dyslexia: dysphonetic dyslexia (DD), dyseidetic, mixed and besides a fourth group defined nonspecific reading delay (NSRD). The subtypes are identified by an algorithm that considers the reading quotient and the percentage of errors in the spelling test. Thanks to this precise clinical distinction, it was possible to observe that the dysphonetic subjects compared to the subjects with nonspecific reading delay had significant higher activity in delta and theta bands in the frontal, central, and parietal areas bilaterally. The hypothesis that there is a timing defect at the basis of dyslexia is confirmed by the study of the effective and directed connectivity. Two very important areas perform as hubs in the information flow: one is the left calcarine sulcus, which is more active in the DD group, and the second is the left rolandic operculum, which is more active in the NSRD group. In the DD group, the calcarine sulcus is sending information to the right postcentral gyrus, the left paracentral gyrus, the right angular gyrus, and the right supplementary motor area. This flow of information occurs in almost all frequency bands, including delta and theta band. Slow connections may indicate less efficient or even 
pathological information flow. In conclusion, dyslexia can be defined, from a psychophysiological point of view, as a cerebral dysregulation in all frequency bands that affects programming, planning, fast processing, and integration of sensory information. All these phenomena occur at different levels of the central nervous system and at different times.

\section{References}

Bode, E. (1973). Developmental dyslexia: A diagnostic approach based on three atypical reading-spelling patterns. Developmental Medicine \& Child Neurology, 15(5), 663-687. https://doi.org/10.1111/j.1469-8749.1973.tb05180.x

Chiarenza, A. C. (2010). Word searches in L1 and L2 Italian conversation: Re-establishing intersubjectivity (Unpublished doctoral dissertation). University of Illinois, UrbanaChampaign.

\section{Regulating Posttraumatic Stress Disorder Symptoms with Neurofeedback: Regaining Control of the Mind \\ Ruth Lanius \\ University of Western Ontario, Ontario, Canada}

Objective. The default mode network (DMN) and salience network (SN) have been shown to be dysregulated in posttraumatic stress disorder (PTSD). Restoring aberrant connectivity within these networks with electroencephalogram neurofeedback (EEG-NFB) has been shown previously to be associated with decreased PTSD symptoms. Here, we conducted a double-blind, sham-controlled randomized clinical trial of alpha-rhythm EEG-NFB in participants with PTSD $(n=36)$ over 20 weeks. Our aim was to provide mechanistic evidence underlying clinical improvements by examining changes in network connectivity via fMRI.

Methods. We randomly assigned participants with a primary diagnosis of PTSD to either the experimental group $(n=18)$ or sham-control group $(n=18)$. We collected resting-state fMRI scans pre- and post-NFB intervention, for both the experimental and shamcontrol PTSD groups, where we additionally compared baseline connectivity measures pre-NFB to age-matched healthy control participants $(n=36)$.

Results. We found significantly decreased PTSD severity scores in the experimental NFB group only, when comparing post-NFB $(d=0.91)$ and 3-month follow-up scores $(d=1.05)$ to baseline measures. Interestingly, we found evidence to suggest a normalization of DMN and SN connectivity post-NFB in the experimental group only. Both decreases in PTSD severity and NFB performance were correlated to decreased insula connectivity with the $\mathrm{SN}$ in the experimental group. Critically, $61.1 \%$ of individuals in the experimental group no longer met criteria for PTSD after treatment, in comparison to $33.3 \%$ in the sham-control group.

Conclusion. The current study shows mechanistic evidence for therapeutic changes in DMN and SN connectivity that are known to be associated with PTSD psychopathology. The current intervention appears well tolerated with no participant dropouts and may prove to be a highly beneficial adjunct treatment for PTSD.

\section{PLENARY SESSION PRESENTATIONS}

\section{Automatic De-artifacting in Normative qEEG Databases: Does Reality Follow the Hype? Marco Rotonda ${ }^{1}$ and Antonia Thelen ${ }^{2}$ ${ }^{1}$ eemagine Imaging Medical Solutions $\mathrm{GmbH}$, Berlin, Germany ${ }^{2}$ ANT neuro $\mathrm{GmbH}$, Berlin, Germany}

Introduction. Advances in the field of automated deartifacting algorithms for electroencephalographic (EEG) signals have provided powerful tools to significantly speed up data processing (Blum, Jacobsen, Bleichner, \& Debener, 2019; Delorme, Sejnowski, \& Makeig, 2007; Makeig, Bell, Jung, \& Sejnowski, 1996; Mullen et al., 2015; Nolan, Whelan, \& Reilly, 2010). Nonetheless, the efficacy of such, sometimes preimplemented algorithms in marketed solutions for qEEG analysis still needs to be addressed. Here, we directly compared the performance of automatic algorithms implemented in different normative qEEG databases (DBs) currently available on the market (Neuroguide, qEEG-Pro, and iMediSync) with a manual artifact-rejection approach.

Materials \& Methods (1). Raw, eyes-closed restingstate EEG data from four exemplar subjects were fed into the preimplemented, automated artifactdetection, and rejection or correction pipelines of the three DBs. Additionally, manual artifact-detection and rejection was performed separately. Thereafter, the absolute power values for five different frequency bands (FBs; delta, theta, alpha, beta1, beta2) were computed at each electrode position (19 channels, $10 / 20$ layout) within each DB. The resulting values were assessed in a mixed-design ANOVA (withinsubject factors: 2 (automated cleaning vs. manual rejection) $\times 3$ (DBs) x 5 (FBs); between-subject factor: electrode).

Results (1). Results suggested significant effects between DBs, which were driven by the amplitude of the absolute power values computed. Specifically, we found a main effect (ME) for DB and FB. 
Additionally, we found significative interactions between DB $x$ Cleaning, Cleaning $x$ FBs and DB $x$ Cleaning $\times$ FBs.

Interim Conclusion (1). Taken alone, these results provide striking evidence that artifact-detections algorithm implemented in the selected DBs hugely impact data interpretation and can hence eventually diverge diagnostic interpretations when absolute power values are taken into consideration.

Materials \& Methods (2). In order to correct for normalization biases across DB outputs in the current analyses, absolute power measures were transformed into $z$-score values across the whole dataset. Hereby, we sought to eliminate false positives in our statistical evaluation due to the different implementation of the power computation algorithms between DBs (White, 2003).

Results (2). The mixed-design ANOVA suggested significant differences as a function of Electrode (DB $x$ Electrode; FBs $x$ Electrode).

Interim Conclusion (2). These results suggest that normalization processes can effectively reduce erroneous statistical differences due to differential implementation amplitude computations. Nonetheless, significant Electrode x DB interactions persist.

General Discussion. Taken together, these preliminary findings underline the existent pitfalls regarding de-artifacting algorithms across DBs. Specifically, differential implementation of absolute power value computation greatly diverges between the present DBs investigated (similar: White, 2003; contrary: Thatcher \& Lubar, 2009, and Keizer, 2018). Moreover, normalization of the data still produced significant differences across DBs.

Conclusion, Open Questions, and Future Directions. Several open questions remain to be addressed. First, the impact of differential cohorts employed between DBs for normalization processes has not been directly assessed in the present study. Second, whether automatic de-artifacting algorithms consistently outperform manual data cleaning needs to be further investigated. Third, the qualitative differences of the automatic de-artifacting algorithms employed by different DBs need further comparisons. We are aware of the limited sample size and aim at increasing the sample size.

\section{References}

Blum, S., Jacobsen, N. S. J., Bleichner, M. G., \& Debener, S. (2019). A Riemannian modification of artifact subspace reconstruction for EEG artifact handling. Frontiers in Human Neuroscience, $13, \quad 141 . \quad$ https://doi.org/10.3389 /fnhum.2019.00141

Delorme, A., Sejnowski, T., \& Makeig, S. (2007). Enhanced detection of artifacts in EEG data using higher-order statistics and independent component analysis. Neurolmage, 34(4), 1443-1449. https://doi.org/10.1016/j.neuroimage.2006.11.004

Keizer, A. W. (2018). A systematic comparison and evaluation of four major QEEG databases. International Journal of Psychophysiology, 131(Suppl.), S2-S3. https://doi.org /10.1016/j.ijpsycho.2018.07.011

Makeig, S., Bell, A. J., Jung, T.-P., \& Sejnowski, T. J. (1996). Independent component analysis of electroencephalographic data. In D. Touretzky, M. Mozer, \& M. Hasselmo (Eds.), Advances in neural information processing systems (pp. 145151). Cambridge MA: MIT Press.

Mullen, T. R., Kothe, C. A. E., Chi, Y. M., Ojeda, A., Kerth, T. Makeig, S., ... \& Cauwenberghs, G. (2015). Real-time neuroimaging and cognitive monitoring using wearable dry EEG. IEEE Transactions on Biomedical Engineering, 62(11), 2553-2567. https://doi.org/10.1109/tbme.2015.2481482

Nolan, H., Whelan, R., \& Reilly, R. B. (2010). FASTER: Fully automated statistical thresholding for EEG artifact rejection. Journal of Neuroscience Methods, 192(1), 152-162. https://doi.org/10.1016/j.jneumeth.2010.07.015

Thatcher, R. W., \& Lubar, J. F. (2009). History of the scientific standards of QEEG normative databases. Introduction to Quantitative EEG Neurofeedback, 2009, 29-59. https://doi.org/10.1016/b978-0-12-374534-7.00002-2

White, J. N. (2003). Comparison of QEEG reference databases in basic signal analysis and in the evaluation of adult ADHD. Journal of Neurotherapy, 7(3-4), 123-169. https://doi.org /10.1300/j184v07n03_06

\section{Combining Bio- and Neurofeedback with Virtual Reality in the Treatment of Anxiety: Current Applications, Research Findings, and Future Directions \\ Jeff Tarrant \\ NeuroMeditation Institute, Eugene, Oregon, USA}

Virtual reality (VR) is a computer-generated environment that simulates a realistic experience in three-dimensional space. While this technology has been primarily associated with the video game industry, it is increasingly being utilized as a clinical intervention for a variety of medical and mental health concerns (Rizzo \& Koenig, 2017). As a mental health intervention, VR has primarily been used as a sophisticated addition to exposure therapy in the treatment of phobias (e.g., fear of heights, flying, etc.; Lamson, 1994; Rothbaum et al., 1995).

In part, the success of these programs appears to be based on the understanding that immersive environments, such as those provided in VR, can generate strong feelings of "presence" (Riva, Waterworth, Waterworth, \& Mantovani, 2011; Riva \& 
Waterworth, 2014; Waterworth \& Riva, 2014; Waterworth, Waterworth, Mantovani, \& Riva, 2010). "Presence," in this context, is defined as the subjective feeling of being in another place and is a crucial element in exposure and distraction-based therapies. Because VR is immersive, it should not be surprising that this format can provide more presence than two-dimensional scenes (Chirico et al., 2017).

Based on this understanding, some VR programs have created specific environments designed to induce a relaxation response. These programs tend to layer elements that have demonstrated effectiveness in stress management programs, such as exposure to nature, mindfulness, and soothing music (Tarrant, Viczko, \& Cope, 2018). Not surprisingly, some companies have created VR and augmented reality (AR) systems that interface with various bio- and neuromodulation techniques, including EEG and HRV. Early research suggests that this combination may provide a powerful tool to assist those working to manage clinical anxiety disorders.

In this presentation, we will explore consumer and clinical programs that combine bio/neurofeedback with both VR and AR as intervention for chronic stress and anxiety. The presenter will summarize results and limitations of four original studies demonstrating the impact of therapeutic VR/AR experiences on brainwave activity, anxiety, and mood states. These studies have demonstrated that a brief mindfulness in nature VR experience can significantly shift brainwave patterns associated with the stress response while a control condition does not (Tarrant et al., 2018). Other results have demonstrated significant increases in subjective feelings of calm and happiness while significantly reducing feelings of tension, anger, and depression. Early results combining VR and $A R$ with a consumer grade EEG headband have demonstrated shifts in frontal asymmetry (Tarrant \& Cope, 2018), as well as increased motivation and likelihood of future use. In addition to clarifying the current state of this work, the presentation will explore future directions, integration of VR with clinical neurofeedback systems, and strategies for using consumer-based programs as adjunctive training for specific client populations.

\section{References}

Chirico, A., Cipresso, P., Yaden, D. B., Biassoi, F., Riva, G., \& Gaggioli, A. (2017). Effectiveness of immersive videos in inducing awe: An experimental study. Scientific Reports, 7, 1218. https://doi.org/10.1038/s41598-017-01242-0

Lamson, R. J. (1994). Virtual therapy of anxiety disorders. CyberEdge Journal, 4(2), 1, 6-8.
Riva, G., \& Waterworth, J. A. (2014). Being present in a virtual world. In M. Grimshaw (Ed.), The Oxford Handbook of Virtuality (pp. 205-221). New York, NY: Oxford University Press. https://doi.org/10.1093/oxfordhb/9780199826162.013.015

Riva, G., Waterworth, J. A., Waterworth, E. L., \& Mantovani, F. (2011). From intention to action: The role of presence. New Ideas in Psychology, 29(1), 24-37. https://doi.org/10.1016 /j.newideapsych.2009.11.002

Rizzo, A. S., \& Koenig, S. T. (2017). Is clinical virtual reality ready for primetime? Neuropsychology, 31(8), 877-899. https://doi.org /10.1037/neu0000405

Rothbaum, B. O., Hodges, L., Kooper, R., Opdyke, D., Williford, J. S., \& North, M. (1995). Effectiveness of computer-generated (virtual reality) graded exposure in the treatment of acrophobia. The American Journal of Psychiatry, 152(4), 626-628. https://doi.org/10.1176/ajp.152.4.626

Tarrant, J., Viczko, J., \& Cope, H. (2018). Virtual reality for anxiety reduction demonstrated by quantitative EEG: A pilot study. Frontiers in Psychology, 9, 1280. https://doi.org/10.3389 /fpsyg.2018.01280

Tarrant, J., \& Cope, H. (2018). Combining frontal gamma asymmetry neurofeedback with virtual reality: A proof-ofconcept case study. NeuroRegulation, 5(2), 57-67. https://doi.org/10.15540/nr.5.2.57

Waterworth, J. A., Waterworth, E. L., Mantovani, F., \& Riva, G. (2010). On feeling (the) present: An evolutionary account of the sense of presence in physical and electronically-mediated environments. Journal of Consciousness Studies, 17(1-2), 167-188.

Waterworth, J., \& Riva, G., (2014). Feeling present in the physical world and in computer-mediated environments. Basingstoke: London, UK: Palgrave Macmillan. https://doi.org/10.1057 /9781137431677

\section{Counteracting Our Current Happiness Deficit by "Happitation" Via Neurofeedback Jonathan Cowan ${ }^{1}$ and Estate Sokhadze ${ }^{2}$ \\ ${ }^{1}$ Peak Achievement Training, Goshen, Kentucky, USA \\ ${ }^{2}$ University of South Carolina School of Medicine, Greenville, South Carolina, USA}

There is a basic truth we often forget: We can create happiness directly from within ourselves. Relatively few people, except for those exposed to positive psychology techniques, know how to control the transition between an ordinary mood and a very happy one.

Learning to do this well and sustain it is very valuable in general, but particularly so now, when the COVID19 crisis has taken away so many of our sources of happiness and other positive feelings-and may continue to do so indefinitely. The best way to deal with this "happiness deficit" is to learn to make the neuropsychological transition in brain function to activate the brain's reward systems, based primarily on increasing dopamine function. This self-control can be learned by generalizing a particular type of neurofeedback training-enhancing the Neureka! rhythm-to other situations in our life by a simple practice we call "Happitation." This transition starts with a deep inhalation, followed by coupling the 
exhalation with the practice of the skill of enhancing Neureka! output, remembered from Neureka! and Focus training sessions on the Happi Trainer. By repeating this a number of times, a feeling of extreme happiness can be created and sustained, even while other activities are being performed.

We believe that control of this transition is natural and based primarily on changing basic biological systems, which are more powerful sustainers of mood than psychological practices, although these certainly can be taught together. Our studies of neurofeedback combining single-pointed Focus via our InhibitAll protocol with enhancing clarified $40 \mathrm{~Hz}$. rhythmNeureka!-have shown that we can enhance happiness for at least four months after just twelve 25min sessions of training. We also demonstrated preto posttraining improvements in both attention and memory, which we believe are predominantly due to Neureka! The combination of these three improvements is exactly what would be expected from enhanced dopamine levels in the prefrontal cortex and elsewhere.

This training study built on a group of three experiments which demonstrated a more specific relationship between Neureka! levels and positive feelings, including happiness, love, satisfaction, joy, gratitude, mindfully watching, anticipating something good, and Aha! There were negative relationships between Neureka! and stress, disappointment, and boredom. Counteracting these three are particularly important now.

Neurofeedback trainers should recognize that there is an increased demand for training that will teach people to be happy whenever they want to create it from within themselves. Although this direct teaching of the most important goal in life stated by many Eastern sages has been elusive, recent progress in neurofeedback makes Happitation training very possible on a widespread scale.

It also offers a new therapeutic approach for decreasing the addict's dependence on outside sources for the creation of their happiness. This is a real key to counteracting addiction.

\section{References}

Cowan, J., D., \& Rubik, B. (2009, September). Positive subjective experiences related to clarified gamma brainwave neurofeedback from the prefrontal cortical region of meditators and non-meditators. Presented at the International Society for Neurofeedback and Research (ISNR) 17th Annual Conference, Indianapolis, IN.

Cowan, J., \& Sokhadze, E. (2010, September). Happiness specifically increases a clarified $40 \mathrm{~Hz}$ EEG rhythm used for neurofeedback. Presented at the International Society Neurofeedback Research (ISNR) 18th Annual Conference, Denver, CO.

Cowan, J. D., \& Sokhadze, E. (2011). Prefrontal gamma neurofeedback improves emotional state and cognitive function. Applied Psychophysiology \& Biofeedback, 36, 220.

Cowan, J., \& Blum, K. Finding happiness and activating the Neureka! neurofeedback way: We can return to Neverland. Retrieved from www.thesoberworld.com , pp. 28-29.

Cowan, J., \& Sokhadze, E. (2018). Understanding of mysterious 40 $\mathrm{Hz}$ brain system for attention, learning, and feeling good. Proceedings of the 2018 ISNR Conference. NeuroRegulation 5(4), 159-160. https://doi.org/10.15540/nr.5.4.150

Rubik, B. N. (2011). Neurofeedback-enhanced gamma brainwaves from the prefrontal cortical region of meditators and nonmeditators and associated subjective experiences. The Journal of Alternative and Complementary Medicine, 17(2), 109-115. https://doi.org/10.1089/acm.2009.0191

Sokhadze, E. M., \& Daniels, R. (2016) Effects of prefrontal $40 \mathrm{~Hz}$ centered EEG band neurofeedback on emotional state and cognitive functions in adolescents. Adolescent Psychiatry, 6(2), 116-129. https://doi.org/10.2174 /2210676606666161025115616

Sokhadze, E. M., Cowan, J., Wang, Y., Casanova, M., Lamina, E., \& Tasman, A. (2016) Effects of prefrontal neurofeedback on perceived emotional state and cognitive functioning in adolescents with drug abuse history. NeuroRegulation, 3(4), 191. https://doi.org/10.15540/nr.3.4

Sokhadze, E. (2012). Peak performance training using prefrontal EEG biofeedback. Biofeedback, 39, 7-15.

\section{Exploratory Study of Loreta Z-Scored}

Neurofeedback and Homeostatic Learning in a Group with Mild Traumatic Brain Injury (MTBI) and Post-Concussion Syndrome (PCS) Rex Cannon ${ }^{1}$, Nanja Schorel, and Rianne Schorel ${ }^{2}$ ${ }^{1}$ SPESA Research Institute, Knoxville, Tennessee, USA

${ }^{2}$ Move the Brain, Zeist, Netherlands

Introduction. Persistent symptoms after mild traumatic brain injury (mTBI) are often debilitating to the individual. This postconcussion syndrome (PCS) can impact social, emotional, and functional domains, thereby increasing financial and resource burdens on healthcare systems and individuals. Debate continues concerning the etiology of PCS and how to best diagnose and treat the symptoms that can persist for months to years after the initial injury (D'Souza et al., 2015; Datta, Pillai, Rao, Kovoor, \& Chandramouli, 2009; Duff, 2004; Iverson, 2019; Kennedy, Quinn, Tumilty, \& Chapple, 2017; Kenzie et al., 2018; Khong, Odenwald, Hashim, \& Cusimano, 2016). Recent data have explored cortisol in relation to $\mathrm{mTBI}$ and continued symptoms, with notable differences in these populations.

Methods. This study examines data for 17 clients (5 male), mean age 34.88, SD 11.07 with a diagnosis of PCS according to ICD-10 criteria (World Health Organization, 1992) that completed a holistic program with neurofeedback as a primary intervention. 
LORETA $z$-score training was conducted twice per day for 10 days in conjunction with other program components (Cannon et al., 2007; Cannon et al., 2014; Thatcher, 2000; Thatcher et al., 2001). Program measures for outcomes were morning cortisol levels, the Dutch version of brief symptom inventory (BSI) and LORETA electrical neuroimaging. We utilized paired comparisons to contrast data across three time points. Nonparametric statistical analyses of functional LORETA images were performed for each contrast using voxel-wise randomization for within subject contrasts (paired $t$ tests) with a threshold $p<.05$.

Results. For this group of clients there was a decrease in morning cortisol levels at 10-day posttraining and at 30-day follow-up. Clinical scales on the BSI showed significant decreases at both 10day and 30-day follow-up measures. LORETA contrasts showed significant changes in Brodmann Areas (BA) 9, 36, 47, 19 and 6. Cortical-cortisol associations showed significant elevations in delta at BA 9 pretraining. While at post and follow-up there appears to be a more diffuse integration of corticocortisol relations.

Conclusions. Holistic approaches with neurofeedback may offer the potential to aid individuals in reducing symptoms and improving functional domains, as well as improving approach related behaviors and executive functions as associated with frontal lobes. Larger sample size and randomized controlled trials are goals for future research as well as developing standard procedures for discovery of bio/psychometric combinations to aid in differentiating PCS from other syndromes.

\section{References}

Cannon, R., Lubar, J., Congedo, M., Thornton, K., Towler, K., \& Hutchens, T. (2007). The effects of neurofeedback training in the cognitive division of the anterior cingulate gyrus. International Journal of Neuroscience, 117(3), 337-357. https://doi.org/10.1080/00207450500514003

Cannon, R. L., Baldwin, D. R., Diloreto, D. J., Phillips, S. T., Shaw, T. L., \& Levy, J. J. (2014). LORETA neurofeedback in the precuneus: Operant conditioning in basic mechanisms of selfregulation. Clinical EEG and Neuroscience, 45(4), 238-248. https://doi.org/10.1177/1550059413512796

D'Souza, M. M., Trivedi, R., Singh, K., Grover, H., Choudhury, A., Kaur, P., ... Tripathi, R. P. (2015). Traumatic brain injury and the post-concussion syndrome: A diffusion tensor tractography study. Indian Journal of Radiology and Imaging, 25(4), 404414. https://doi.org/10.4103/0971-3026.169445

Datta, S. G. S., Pillai, S. V., Rao, S. L., Kovoor, J. M. E., \& Chandramouli, B. A. (2009). Post-concussion syndrome: Correlation of neuropsychological deficits, structural lesions on magnetic resonance imaging and symptoms. Neurology India, 57(5), 594-598. https://doi.org/10.4103/0028-3886.57810
Duff, J. (2004). The usefulness of quantitative EEG (QEEG) and neurotherapy in the assessment and treatment of postconcussion syndrome. Clinical EEG and Neuroscience, 35(4), 198-209. https://doi.org/10.1177/155005940403500410

Iverson, G. L. (2019). Network analysis and precision rehabilitation for the post-concussion syndrome. Frontiers in Neurology, 10, 489. https://doi.org/10.3389/fneur.2019.00489

Kennedy, E., Quinn, D., Tumilty, S., \& Chapple, C. M. (2017). Clinical characteristics and outcomes of treatment of the cervical spine in patients with persistent post-concussion symptoms: A retrospective analysis. Musculoskeletal Science and Practice, 29, 91-98. https://doi.org/10.1016 /j.msksp.2017.03.002

Kenzie, E. S., Parks, E. L., Bigler, E. D., Wright, D. W., Lim, M. M., Chesnutt, J. C., ... Wakeland, W. (2018). The dynamics of concussion: Mapping pathophysiology, persistence, and recovery with causal-loop diagramming. Frontiers in Neurology, 9, 203. https://doi.org/10.3389/fneur.2018.00203

Khong, E., Odenwald, N., Hashim, E., \& Cusimano, M. D. (2016). Diffusion tensor imaging findings in post-concussion syndrome patients after mild traumatic brain injury: A systematic review. Frontiers in Neurology, 7, 156. https://doi.org/10.3389 /fneur.2016.00156

Thatcher, R. W. (2000). EEG operant conditioning (biofeedback) and traumatic brain injury. Clinical Electroencephalography, 31(1), 38-44. https://doi.org/10.1177/155005940003100110

Thatcher, R. W., Biver, C., Gomez, J. F., North, D., Curtin, R., Walker, R. A., \& Salazar, A. (2001). Estimation of the EEG power spectrum using MRI T(2) relaxation time in traumatic brain injury. Clinical Neurophysiology, 112(9), 1729-1745. https://doi.org/10.1016/s1388-2457(01)00609-5

World Health Organization. (1992). The ICD-10 classification of mental and behavioral disorders: Clinical descriptions and guidelines. Geneva, Switzerland: World Health Organization.

Infraslow Neurofeedback, the Latest Research

Mark Llewellyn ${ }^{1}$, Tyson Perez ${ }^{2}$, and Jerin Mathew ${ }^{2}$ ${ }^{1}$ Neurofeedback Services of NY, New York, New York, USA

${ }^{2}$ University of Otago, Dunedin, New Zealand

Infraslow fluctuation training (ISF) was recently tested in a randomized, double-blind, placebo-controlled study that explored the therapeutic effects on obese women with food addiction (Leong et al., 2018). The success of that project heralded an increase in interest in and studies on ISF training recently. This presentation will summarize the research results of three current studies, one on bipolar ISF training that involves two channels (Smith, Collura, Ferrera, \& de Vries, 2014) and a 19-channel variation: ISF SLORETA.

In our most recently completed study (in press) we compare the impact of ISF bipolar training and Sensory Motor Rhythm (SMR) neurofeedback on the autonomic nervous system (ANS). This randomized study is the first to test a proposed mechanism of action for distinct forms of neurofeedback training. We used peripheral biofeedback devices to measure the autonomic impact of both interventions on subjects with anxiety disorders. 
Additionally, we will report on two studies of another form of ISF training: ISF sLORETA training. In collaboration with Dr. Dirk De Ridder, and in consideration of the current neuroscience of pain, we have developed a neurofeedback protocol for chronic pain. This innovative design is currently being tested in another "Gold Standard" (randomized, doubleblind, sham-controlled) study. The protocol and research design have been published in NeuroRegulation (Mathew, Adhia, Smith, De Ridder, \& Mani, 2020). We will report on phase one of a three-phase research design that will involve approximately 100 subjects upon completion.

The final study we report on in this presentation is ISF sLORETA for affective disorders. Recent literature has proposed a central autonomic network (CAN) in cortex that impacts autonomic nervous system function. It theorizes that the CAN is centrally involved in all aspects of human behavior and is therefore a critical component of psychopathology (Beissner, Meissner, Bär, \& Napadow, 2013; Thayer \& Lane, 2000). Also a randomized, double-blind, sham-controlled study, the affective disorder project targets both the central hubs of sympathetic and parasympathetic function in cortex. These are primary ROIs within the CAN structure. With an innovative design the ISF SLORETA protocol attempts to regulate these hubs by assuming disordered activation patterns.

The functional architecture of the brain is coordinated by infraslow frequencies. ISF coordinates both the connections within and decoupling between active behavioral networks (Palva \& Palva, 2012). This slow regime, described as $<0.1 \mathrm{~Hz}$, was first linked with behavior by Nina Aladjalova in the 1950s in the Soviet Union. Aladjalova associated infraslow activity with parasympathetic, reparative response (Aladjalova, 1957). Recently, there has been a dramatic increase in research of human behaviour and the infraslow frequencies. Studies of ISF and ADHD, pain, and sleep, to name just a few, have been published (Alshelh et al., 2016; Broyd, Helps, \& Sonuga-Barke, 2011; Lecci et al., 2017).

\section{References}

Aladjalova, N. A. (1957). Infra-slow rhythmic oscillations of the steady potential of the cerebral cortex. Nature, 179(4567), 957-959. https://doi.org/10.1038/179957a0

Alshelh, Z., Di Pietro, F., Youssef, A. M., Reeves, J. M., Macey, P. M., Vickers, E. R., ... Henderson, L. A. (2016). Chronic neuropathic pain: It's about the rhythm. The Journal of Neuroscience, 36(3), 1008-1018. https://doi.org/10.1523 /jneurosci.2768-15.2016

Beissner, F., Meissner, K., Bär, K.-J., \& Napadow, V. (2013). The autonomic brain: An activation likelihood estimation metaanalysis for central processing of autonomic function. The
Journal of Neuroscience, 33(25), 10503-10511. https://doi.org/10.1523/jneurosci.1103-13.2013

Broyd, S. J., Helps, S. K., \& Sonuga-Barke, E. J. S. (2011). Attention-induced deactivations in very low frequency EEG oscillations: Differential localisation according to ADHD symptom status. PLoS ONE, 6(3), e17325. https://doi.org/10.1371/journal.pone.0017325

Lecci, S., Fernandez, L. M. J., Weber, F. D., Cardis, R., Chatton, J.-Y., Born, J., \& Lüthi, A. (2017). Coordinated infraslow neural and cardiac oscillations mark fragility and offline periods in mammalian sleep. Science Advances, 3(2) ), e1602026. https://doi.org/10.1126/sciadv.1602026

Leong, S. L., Vanneste, S., Lim, J., Smith, M., Manning, P., \& De Ridder, D. (2018). A randomised, double-blind, placebocontrolled parallel trial of closed-loop infraslow brain training in food addiction. Scientific Reports, 8(1), 11659. https://doi.org/10.1038/s41598-018-30181-7

Mathew, J., Adhia, D. B., Smith, M. L., De Ridder, D., \& Mani, R. (2020). Protocol for a pilot randomized sham-controlled clinical trial evaluating the feasibility, safety, and acceptability of infraslow electroencephalography neurofeedback training on experimental and clinical pain outcomes in people with chronic painful knee osteoarthritis. NeuroRegulation, 7(1), 30-44. https://doi.org/10.15540/nr.7.1.30

Palva, J. M., \& Palva, S. (2012). Infra-slow fluctuations in electrophysiological recordings, blood-oxygenation-leveldependent signals, and psychophysical time series. Neurolmage, 62(4), 2201-2211. https://doi.org/10.1016 /j.neuroimage.2012.02.060

Smith, M. L., Collura, T. F., Ferrera, J., \& de Vries, J. (2014). Infraslow fluctuation training in clinical practice: A technical history. NeuroRegulation, 1(2), 187-207. https://doi.org/10.15540 /nr.1.2.187

Thayer, J. F., \& Lane, R. D. (2000). A model of neurovisceral integration in emotion regulation and dysregulation. Journal of Affective Disorders, 61(3), 201-216. https://doi.org/10.1016 /S0165-0327(00)00338-4

\section{Neurofeedback in Healthy Elderly at Risk of Cognitive Decline \\ Mauricio Gonzalez-Lopez}

Sociedad Mexicana de Bio y Neurofeedback, Juriquilla, Querétaro, Mexico

Age is the main risk factor for the incidence of neurocognitive disorders. Past research has shown that quantitative electroencephalography (qEEG) might be a good tool to assess the risk of developing future cognitive decline in a healthy elderly population, and specifically an excess of theta activity $(4.0-8.0 \mathrm{~Hz})$ has been observed (Prichep et al., 2006, Prichep, 2007). Based on this rationale we have explored whether an excess of theta activity, even when all neuropsychological variables seem normal, might discriminate in neurobiological terms between two populations of healthy elderly. For this reason, we explored with several neurobiological tools, such as event-related potentials and magnetic resonance imaging, whether these two populations would differ, in order to provide: a) a rationale for a neurofeedback treatment and b) neurophysiological tools to assess the efficacy of a specific neurofeedback protocol. Therefore, with this two-fold objective in mind, we 
present two sets of comparisons: 1) the results of the neurophysiological comparison between a group considered to be at risk of cognitive decline (i.e., those that presented an excess of theta activity compared to a normative database) and a control group with normal EEG; and 2) the neuropsychological results and EEG changes after a neurofeedback protocol that aimed to normalize these theta excesses, as well as the comparison with a placebo group. We observed a difference in psychophysiological terms between the Risk group (before treatment) and the Control group, when considering biological measures but not psychometric and neuropsychological tools. Moreover, we observed differences posttreatment between the Neurofeedback and the Placebo (Sham) group. We also present preliminary results of a sLORETA treatment, which aimed to normalize the most abnormal current sources in the theta band. This evidence contributes to the idea that normalizing an abnormal excess of theta activity might have beneficial effects on cognition in this elderly subgroup, and quantitative and normative EEG might be a low-cost and viable tool for the early identification of risk of cognitive decline.

Acknowledgements. Catalina Alatorre, Susana Castro, Sergio Sánchez, Francisca Salcedo, Carolina Villada, Rodrigo Flores, Monserrat Palacios, Saulo Hernández, and Hector Belmont for technical support and Teresa Alvarez for personal assistance. This work was funded by grants IN225414 and IN200817 from PAPIIT DGAPA, as well as scholarship 608617 from CONACYT.

\section{References}

Alatorre-Cruz, G. C., Silva-Pereyra, J., Fernández, T., RodríguezCamacho, M. A., Castro-Chavira, S. A., \& Sanchez-Lopez, J. (2018). Effects of age and working memory load on syntactic processing: An event-related potential study. Frontiers in Human Neuroscience, 12, 185. https://doi.org/10.3389 /fnhum.2018.00185

Becerra, J., Fernández, T., Roca-Stappung, M., Díaz-Comas, L., Galán, L., Bosch, J., ... Harmony, T. (2012). Neurofeedback in healthy elderly human subjects with electroencephalographic risk for cognitive disorder. Journal of Alzheimer's Disease, 28(2), 357-367. https://doi.org/10.3233/jad-2011-111055

Castro-Chavira, S. A., Barrios, F. A., Pasaye, E. H., Alatorre-Cruz, G. C., \& Fernández, T. (2016). Compensatory larger cortical thickness in healthy elderly individuals with electroencephalographic risk for cognitive decline. NeuroReport, 27(9), 710-715.

Prichep, L. S., John, E. R., Ferris, S. H., Rausch, L., Fang, Z., Cancro, R., ... Reisberg, B. (2006). Prediction of longitudinal cognitive decline in normal elderly with subjective complaints using electrophysiological imaging. Neurobiology of Aging, 27(3), $\quad 471-481 . \quad$ https://doi.org/10.1016 /j.neurobiolaging.2005.07.021

Prichep, L. S. (2007). Quantitative EEG and electromagnetic brain imaging in aging and in the evolution of dementia. Annals of the New York Academy of Sciences, 1097(1), 156-167. https://doi.org/10.1196/annals.1379.008

Sánchez-Moguel, S. M., Alatorre-Cruz, G. C., Silva-Pereyra, J., González-Salinas, S., Sanchez-Lopez, J., Otero-Ojeda, G. A., \& Fernández, T. (2018). Two different populations within the healthy elderly: Lack of conflict detection in those at risk of cognitive decline. Frontiers in Human Neuroscience, 11, 658. https://doi.org/10.3389/fnhum.2017.00658

Noninvasive Brain Stimulation Interventions to Elevate Neurofeedback Outcomes - PBM, TDCS, TACS, TMS, and Others

Lew Lim

Vielight, Inc., Toronto, Ontario, Canada

Hypothesis. Noninvasive brain stimulation (NIBS) interventions can potentially elevate neurofeedback (NFB) outcomes. They include photobiomodulation (PBM) and transcranial electrical stimulation (TES) methods, especially transcranial direct current stimulation (tDCS) and alternating current stimulation (tACS); as well as magnetic-based transcranial magnetic stimulation (TMS). However, up-to-date objective comparative analyses are needed for informed decisions.

Supporting Evidence to Date. Literature are largely inconclusive, pointing to the need for more investigations. TMS and to a lesser extent, tDCS have credible evidence to indicate as treatment of depression, but there is little consensus for other neurological and neuropsychiatric conditions (Lefaucheur et al., 2014). A reason is the lack of understanding the mechanisms of action of these modalities (Terranova et al., 2019). In the case of PBM, the mechanisms are clearer but has relatively lighter empirical data (Giordano et al., 2017). However, emerging evidence demonstrate consistent frequency-dependent response from the brain to PBM (Zomorrodi, Loheswaran, Pushparaj, \& Lim, 2019). New clues are provided in the modulation of the default mode network (DMN; Saltmarche, Naeser, Ho, Hamblin, \& Lim, 2017; Zomorrodi et al., 2019), which points to networks for future PBM research.

Methods. The method here is combines literature review with controlled studies data. An ongoing study is investigating healthy participants treated with PBM induced at an oscillation of $10 \mathrm{~Hz}$ to the default mode network versus alternatives. Another investigates a larger variety of oscillations from $0 \mathrm{~Hz}$ to $200 \mathrm{~Hz}$, along with antiphase inductions between selected regions of the brain.

Results. The new PBM results will be compared to earlier PBM as well as tDCS, tACS, and TMS data. This would lead to further understanding of PBM 
compared to or combined with other NIBS interventions.

Conclusions. The findings from the subject investigations will be promising in advancing the understanding the various NIBS to potentially elevate NFB practice outcomes.

\section{References}

Giordano, J., Bikson, M., Kappenman, E. S., Clark, V. P., Coslett, H. B., Hamblin, M. R., ... Calabrese, E. (2017). Mechanisms and effects of transcranial direct current stimulation. DoseResponse, 15(1), 1-22. https://doi.org /10.1177\%2F1559325816685467

Lefaucheur, J. P., André-Obadia, N., Antal, A., Ayache, S. S., Baeken, C., Benninger, D. H., ..., Garcia-Larrea, L. (2014). Evidence-based guidelines on the therapeutic use of repetitive transcranial magnetic stimulation (rTMS). Clinical Neurophysiology. 125(11), 2150-2206. https://doi.org/10.1016 /j.clinph.2014.05.021

Saltmarche, A. E., Naeser, M. A., Ho, K. F., Hamblin, M. R., \& Lim, L. (2017). Significant improvement in cognition in mild to moderately severe dementia cases treated with transcranial plus intranasal photobiomodulation: Case series report. Photomedicine and Laser Surgery, 35(8), 432-441, https://doi.org/10.1089/pho.2016.4227

Terranova, C., Rizzo, V., Cacciola, A., Chillemi, G., Calamuneri, A., Milardi, D. \& Quartarone A. (2019). Is there a future for noninvasive brain stimulation as a therapeutic tool? Frontiers in Neurology, 9, 1146. https://doi.org/10.3389/fneur.2018.01146

Zomorrodi, R., Loheswaran, G., Pushparaj, A., \& Lim, L. (2019). Pulsed near infrared transcranial and intranasal photobiomodulation significantly modulates neural oscillations: A pilot exploratory study. Scientific Reports, 9, 6309. https://doi.org/10.1038/s41598-019-42693-x

\section{Solving the Mystery: When Children and Adolescents Fail Treatment, Parents Want Answers \\ Ronald Swatzyna and Meredith Hoffman \\ Houston Neuroscience Brain Center, Houston, Texas, USA}

When a child fails to respond as hoped to medication or treatment, their parents want answers. Rarely is neurofeedback their first choice, but it is often their last hope. By the time they consider neurofeedback, most have failed several medication attempts, behavior intervention, and parent training. The reason why so many children fail to get their issues resolved may be due to an inadequate diagnostic process. In the field of mental health, diagnoses are primarily based on the report of symptoms from either the patient, parents, or both, and a psychiatrist's or therapist's observations. A psychiatric diagnosis is currently the most widely used basis for medication and treatment selection; however, the brain is seldom investigated directly as a source of those symptoms. The National Institute of Mental Health (NIMH) Research Domain Criteria Project (RDoC) was created to elicit scientific research into neurological abnormalities that can be linked to psychiatric symptoms for the purpose of predicting medication and treatment response. One such neurological abnormality that has been the focus of many studies over the last three decades is isolated epileptiform discharges (IEDs) in patients without seizures. Zimmerman and Konopka (2014) found that IEDs are associated with greater affective dysregulation and more severe psychiatric symptoms. They found high rates of IEDs have been found in specific diagnostic categories such as panic and anxiety disorders, mood disorders, schizophrenia, eating disorders, personality disorders, and violent behavior. Additionally, high rates of IEDs have been found specific to childhood disorders such as ADHD (Kanazawa, 2014; Lee, Choi, Yoon, \& Bahn, 2015; Milichap, Stack, \& Millichap, 2011; Swatzyna, Tarnow, Roark, \& Mardick, 2017a) and Autism Spectrum Disorder (Chez et al., 2006; Mulligan \& Trauner, 2013; Reinhold, Molloy, \& ManningCourtney, 2005; Swatzyna 2017b; Yasuhara, 2010). We present the findings of our systematic review of the literature and compare them to our crosssectional analysis in order to determine prevalence rates of IEDs within diagnostic categories. Our study found a consistent high prevalence of IEDs specifically for ADHD (majority $>25 \%$ ) and ASD (majority $>59 \%$ ). If children and adolescents have failed multiple medication attempts, and more than one-third of them have IEDs, then an EEG would be justified within the RDoC paradigm. Identification of IEDs is critical to the success of neurofeedback in two ways. First, medications such as stimulants, antidepressants, and antipsychotics all lower seizure threshold and make these brains more unstable/pathologic, thus thwarting neurofeedback efforts. Second, IEDs are either artifacted out or averaged out in making the qEEG brain maps. The foci of the IEDs have to be known so that appropriate neurofeedback protocols can be designed. Just training the background fails to address the primary cause of the child's symptoms. Having the EEG read by a neurologist who is a board-certified encephalographer is not only justified in children and adolescents who have tried and failed past medication attempts but is an ethical responsibility.

\section{References}

Chez, M. G., Chang, M., Krasne, V., Coughlan, C., Kominsky, M., \& Schwartz, A. (2006). Frequency of epileptiform EEG abnormalities in a sequential screening of autistic patients with no known clinical epilepsy from 1996 to 2005. Epilepsy \& Behavior, 8(1), 267-271. https://doi.org/10.1016 /j.yebeh.2005.11.001

Kanazawa, O. (2014). Reappraisal of abnormal EEG findings in children with ADHD: On the relationship between ADHD and 
epileptiform discharges. Epilepsy \& Behavior, 41, 251-256. https://doi.org/10.1016/j.yebeh.2014.09.078

Lee, E. H., Choi, Y. S., Yoon, H. S., \& Bhan, G. H. (2015). Clinical impact of epileptiform discharge in children with attentiondeficit/hyperactivity disorder (ADHD). Journal of Child Neurology, 31(5), 584-588. https://doi.org/10.1177 10883073815604223

Millichap, J. J., Stack, C. V., \& Millichap, J. G. (2011). Frequency of epileptiform discharges in the sleep-deprived electroencephalogram in children evaluated for attentiondeficit disorders. Journal of Child Neurology, 26(1), 6-11. https://doi.org/10.1177/0883073810371228.

Mulligan, C. K., \& Trauner, D. A. (2013). Incidence and behavioral correlates of epileptiform abnormalities in autism spectrum disorders. Journal of Autism and Developmental Disorders, 44(2), 452-458. https://doi.org/10.1007/s10803-013-1888-6

Reinhold, J. A., Molloy, C. A., \& Manning-Courtney, P. (2005). Electroencephalogram abnormalities in children with autism spectrum disorders. Journal of Neuroscience Nursing, 37(3), 136-139. https://doi.org/10.1097/01376517-20050600000003

Swatzyna, R. J., Tarnow, J. D., Roark, A., \& Mardick, J. (2017a). The utility of EEG in attention deficit hyperactivity disorder: A replication study. Clinical EEG and Neuroscience, 48(4), 243245. https://doi.org/10.1177/1550059416640441

Swatzyna, R. J., Tarnow, J. D., Turner, R. P., Roark, A. J., Maclnerney, E. K., \& Kozlowski, G. P. (2017b). Integration of eeg into psychiatric practice: A step toward precision medicine in autism spectrum disorder. Journal of Clinical Neurophysiology, 34(3), 230-235. https://doi.org/10.1097 /wnp.0000000000000365

Yasuhara, A. (2010). Correlation between EEG abnormalities and symptoms of autism spectrum disorder (ASD). Brain \& Development, 32(10), 791-798. https://doi.org/10.1016 /j.braindev.2010.08.010

Zimmerman, E. M., \& Konopka, L. M. (2014). Preliminary findings of single- and multifocused epileptiform discharges in nonepileptic psychiatric patients. Clinical EEG and Neuroscience, 45(4), 285-292. https://doi.org/10.1177 $/ 1550059413506001$

\section{Standardization and Personalized Medicine Using Quantitative EEG in Clinical Settings André Keizer \\ qEEG-Pro, Eindhoven, Netherlands}

Two major trends have been dominant in healthcare in recent years. First, there is a growing consensus that standardization of healthcare procedures and methods can result in improved effectiveness and safety of treatments. There has been a great effort to implement more standardization in diagnosing and treating medical conditions. For example, the World Health Organization initiated the High 5s Project in 2007, which aimed to facilitate the development, implementation, and evaluation of Standard Operating Protocols (SOPs; https://www.who.int /patientsafety/topics/high-5s/en/) for medical treatments in order to increase patient safety. Second, there is increased interest in "personalized medicine," which refers to the tailoring of treatments to individual patients. Personalized medicine has gained considerable traction, mainly as a result of rapid developments in genetic research (Hamburg \& Collins, 2010; Jameson \& Longo, 2015) and novel methods for analyzing big data (Alyass, Turcotte, \& Meyre, 2015). In the current presentation, I will discuss both standardization and personalized medicine in its historical context, how it is applied in different medical fields, and, most importantly, how these trends apply to the field of quantitative EEG (qEEG). There are two important topics that relate to the use of qEEG in clinical practice that need to be addressed in the context of standardization. The first topic relates to the way resting state EEG data is deartifacted. Traditionally, EEGs are de-artifacted manually by trained EEG technicians or EEG researchers. However, in recent years standardized and automated de-artifacting procedures are increasingly being used in scientific research and in clinical practice. The advantages of these procedures over manual de-artifacting will be discussed. The second topic relates to the use of normative databases in order to assess clinically meaningful deviations of a patient's EEG. The results of a systematic comparison between two commonly used qEEG databases show that these databases produce very comparable results, illustrating not only the validity and reliability of both databases, but also the opportunity to move forward to a standardized use of qEEG in clinical practice. The standardization of qEEG analyses enables valid and reliable use of qEEG for diagnostic procedures, to guide personalized treatment protocols and to assess treatment effectiveness. Finally, the standardization of qEEG interpretation as both a diagnostic and treatment selection tool provides an example of how qEEG can merge both personalized medicine and standardization in the treatment of psychological disorders.

\section{References}

Alyass, A., Turcotte, M., \& Meyre, D. (2015). From big data analyses to personalized medicine for all: Challenges and opportunities. BMC Medical Genomics, 8, 33. https://doi.org /10.1186/s12920-015-0108-y

Hamburg, M., A., \& Collins, F. C. S. (2010). The path to personalized medicine. The New England Journal of Medicine, 363(4), 301-304. https://doi.org/10.1056/nejmp1006304

Jameson, J. L., \& Longo, D. L. (2015). Precision medicinepersonalized, problematic, and promising. The New England Journal of Medicine, 372(23), 2229-2234. https://doi.org /10.1056/NEJMsb1503104 


\section{The Reality of the Unreal: Dissociation and}

\section{Dissociative Disorders}

Ainat Rogel and Diana Martinez

Boston NeuroDynamics, Brookline, Massachusetts, USA

Dissociation and dissociative disorders (DD) are among of the most overlooked conditions and challenging therapies, despite their high prevalence. The nature of the symptoms and the fact that the symptoms often accompany many other disorders and are often misdiagnosed are some of the reasons for these claims. Dissociation/DD are among the oldest described psychiatrist conditions. They have a long history and are one of the oldest described psychiatrist conditions. They were first described in the late 18th century, and since then they were accompanied by controversy that extended from professional debates to social, political, and cultural domains. This presentation follows the model that Dissociation/DD are related to trauma and developmental trauma. This model is supported by up-to-date scientific data. However, this model is controversial and not fully accepted. The presentation will discuss a general overview of other relevant models for dissociation/DD. The first goal of this presentation is to unpack the theoretical aspects of dissociation/DD as well as highlighting the clinical aspects of working with people with dissociation/DD. The second goal is to describe subjective assessments as well as objective measurements. The presentation starts with a historical background, models and diagnosis of dissociation/DD and the way they evolved over time as well as pointing out the controversy and the inconsistency over the different models and diagnosis. The presentation continues with the clinical presentations of dissociations/DD and the different diagnosis that are related to it. While the presentation will focus on the relationship between dissociation/DD and trauma, the background will include other modalities. The presentation continues with scientific, clinical, and epidemiological data. The major part of the presentation discusses clinical approaches and challenges of working with clients with dissociation/DD and how to address these unique challenges. Both subjective measurements as well as objective measurements such as EEG and physiological activities will be covered. The presentation concludes with a discussion of future steps clinically as well as in research to improve the outcome of the treatment.

\section{References}

Blihar, D., Delgado, E., Buryak, M., Gonzalez, M., \& Waechter, R. (2020). A systematic review of the neuroanatomy of dissociative identity disorder. European Journal of Trauma \&
Dissociation, 4(3), $100148 . \quad$ https://doi.org/10.1016 /j.ejtd.2020.100148

Liotti, G. (2006). A model of dissociation based on attachment theory and research. Journal of Trauma \& Dissociation. 7(4), 55-73. https://doi.org/10.1300/J229v07n04_04

Loewenstein, R. J. (2018). Dissociation debates: Everything you know is wrong. Dialogues in Clinical Neuroscience, 20(3), 229242. https://doi.org/10.31887/dcns.2018.20.3/rloewenstein

Parry, S., Lloyd, M., \& Simpson, J. (2018). "It's not like you have PSTD with a touch of dissociation": Understanding dissociative identity disorder through first person accounts. European Journal of Trauma \& Dissociation, 2(1), 31-38. https://doi.org/10.1016/j.ejtd.2017.08.002

Spitzer, C., Barnow, S., Freyberger, H. J., \& Grabe, H. J. (2006). Recent developments in the theory of dissociation. World Psychiatry, 5(2), 82-86.

Steele, K., Boon, S., \& van der Hart, O. (2017). Norton series on interpersonal neurobiology. Treating trauma-related dissociation: A practical, integrative approach. New York, NY: W. W. Norton \& Company.

Sar, V. (2011). Epidemiology of dissociative disorders: An overview. Epidemiology Research International, 2011, 404538. https://doi.org/10.1155/2011/404538

van der Kolk, B. A. (2014). The body keeps the score: Brain, mind, and body in the healing of trauma. London, U.K.: Penguin Books.

van der Hart, O., Steele, K., \& Nijenhuis, E. (2017). The treatment of traumatic memories in patients with complex dissociative disorders. European Journal of Trauma \& Dissociation, 1(1), 25-35. https://doi.org/10.1016/j.ejtd.2017.01.008

Zucker, M., Spinazzola, J., Blaustein, M., \& van der Kolk, B. (2006). Dissociative symptomatology in posttraumatic stress disorder and disorders of extreme stress. Journal of Trauma \& Dissociation, 7(1), 19-31. https://doi.org/10.1300 /J229v07n01_03

\section{Visualizing Neurological Decision-Making Pathways to Help Clients Understand Self Ronald Bonnstetter ${ }^{1}$ and Thomas Collura ${ }^{2}$ \\ ${ }^{1}$ TTI Success Insights, Scottsdale, Arizona, USA \\ ${ }^{2}$ BrainMaster Technologies, Inc., Bedford, Ohio, USA}

While neuroscience and mental health professionals acknowledge the role of emotions in decision-making, application of this knowledge is hampered by the lack of a common language and a model that illustrates the potential neurological pathways. By better understanding the brain's decision-making process and the role of emotions in those decisions, we can begin to expose the moment-by-moment dynamics of human behaviors and the role played by precognitive thoughts. Armed with this knowledge, we may be able to help individuals recognize and reflect on decisions in a more logical manner. This presentation will offer insights into how humans react to personal triggers in a conversation, thus exposing underlying precognitive beliefs and related emotions that ultimately lead to our behaviors and decisions. We will highlight the protocols used to generate these modified event-related potentials with a focus on gamma frontal lobe asymmetry as well exposing the asymmetry of Brodmann's areas 9 and 10 as primary 
emotional processing areas and Brodmann's areas 44 and 45 as secondary emotional processing resource. Changes in these Brodmann areas, as a participant processes a new stimulus, will be presented using quantitative analysis and will serve as validation of the resulting parallel SLORETA visual maps.

The ultimate takeaway from this presentation is the creation of a model that illustrates the potential neurological pathways and produces a minimal model that attempts to account for the emotional states and decision processes. When the transitions are viewed in a particular format that accentuates the contributions of the targeted brain locations, a digital code emerges. When a left or right sensory or perceptual area becomes active, then the corresponding portions of the activation model are considered to have a role in the current processing. In addition, the spontaneous categorization of inputs that the model associates with this processing is an example of an adaptive process that exposes an essential survival mechanism that is part of our evolution as a species.

Administering these protocols in real-world contexts, such as during coaching sessions, job interviews, and possibly even in psychotherapeutic milieus (given proper ethical constraints), are promising areas for additional study and promise to impact and potentially expose hidden decision-making mechanisms of the preconscious mind.

\section{References}

Anderson, J. R. (1991). The adaptive nature of human categorization. Psychological Review, 98(3), 409-429. https://doi.org/10.1037/0033-295X.98.3.409

Baranov, S. (2018). Finite state machines and algorithmic state machines. ISBN-13: 978-1-7750917-07

Bonnstetter, R. J., Collura, T., Hebets, D., \& Bonnstetter, B. J. (2013, Winter). Uncovering the belief behind the action. NeuroConnections. Newsletter of The International Society of Neurofeedback and Research and Applied Psychophysiology and Biofeedback Neurofeedback Division. McLean, VA

Collura, T. F., Zalaquett, C., \& Bonnstetter, R. J. (2014). Seeing inside the client's mind. Counseling Today, 57(6), 24-27.

Collura, T. F., Zalaquett, C. P., Bonnstetter, R., Chatters, S. J. (2014). Toward an operational model of decision making, emotional regulation, and mental health impact. Advances in Mind-Body Medicine, 28(4), 4-19.

Davidson, R. J., \& Begley, S. (2012). The emotional life of your brain. New York, NY: Hudson Street Press.

Haidt, J. (2001). The emotional dog and its rational tail: A social intuitionist approach to moral judgment. Psychological Review, 108(4), 814-834. https://doi.org/10.1037/0033295X.108.4.814

Ivey, A. E., Ivey, M. B., \& Zalaquett, C. P. (2014). Intentional interviewing and counseling: Facilitating client development. Belmont, CA: Brooks-Cole.
Michel, C. M., \& Koenig, T. (2018). EEG microstates as a tool for studying the temporal dynamics of whole-brain neuronal networks: A review. Neurolmage, 180(Part B), 577-593. https://doi.org/10.1016/j.neuroimage.2017.11.062

Wiener, N. (1961). Cybernetics-Control and communication in man and machine. Boston, MA: MIT Press.

Neurofeedback in ADHD: Rating the Evidence, APA Guidelines, and a Multicenter Replication Study of qEEG-informed Neurofeedback

Trevor Brown

neuroCare Group, East Melbourne VIC, Australia

Introduction. Precision medicine is uncovering ways to stratify treatment; for example, qEEG recently demonstrated the ability to inform the likelihood of Sertraline versus rTMS response in major depressive disorder (MDD; Wu et al., 2020). Within the neurofeedback field, qEEG assessment can uncover previously unknown sleep-related vigilance regulation difficulties impacting executive function in ADHD cases. The patient's EEG "informs" which standard neurofeedback protocol will be most effective. Thus, qEEG-informed neurofeedback allows personalized intervention, stratifying to the most likely effective protocol. To date, clinical effectiveness data for qEEG-informed neurofeedback have only been published in a small sample of 21 ADHD patients (Arns, Drinkenburg, \& Kenemans, 2012). Recent research (Krepel et al., 2020, presented as the principal study) replicated this effectiveness in a new sample of 114 patients treated with qEEG-informed neurofeedback, from a large multicentric dataset and investigated potential predictors of neurofeedback response.

Methods (of principal study). A sample of 114 patients were included as a replication sample. Patients were assessed with ADHD-RS, PSQI, qEEG and ERPs, then assigned to a standard neurofeedback protocol (SMR, TBR, or SCP neurofeedback) in combination with coaching and sleep hygiene advice. The ADHD-RS and PSQI were assessed at baseline, every 10th session, and at outtake. Response was defined as ADHD-RS > 25\% reduction (R25), > 50\% reduction (R50), and remission. Predictive analyses were focused on predicting remission status.

Results (of principal study). In the current sample, response rates were $85 \%$ (R25), $70 \%$ (R50), and remission was 55\%. Clinical effectiveness was not significantly different from the original 2012 sample. Nonremitters exhibited significantly higher baseline hyperactivity ratings. Women who remitted had significantly shorter P300 latencies and boys who 
remitted had significantly lower individual Alpha Peak Frequencies (iAPF).

Discussion. In the principal study, clinical effectiveness was replicated, suggesting it is possible to assign patients to a protocol based on their individual baseline qEEG to enhance signal-to-noise ratio. Furthermore, remitters had lower baseline hyperactivity scores. Likewise, female remitters had shorter P300 latencies, whereas boys who remitted have a lower iAPF. This latter finding is intriguing, since low IAPF was earlier found to predict nonresponse to MPH (Arns, Gunkelman, Breteler, \& Spronk, 2008), thus offering opportunities to use this biomarker to stratify between treatments. The data suggests initial specificity in treatment allocation, yet further studies are needed to replicate the predictors of neurofeedback remission. A comparison of clinical effectiveness versus RCT efficacy in neurofeedback will lead to a discussion of proposed APA guidelines for rating future evidence (Arns et al., 2020).

\section{References}

Arns, M., Clark, C. R., Trullinger, M., deBeus, R., Mack, M. \& Aniftos, M. (2020). Neurofeedback and attentiondeficit/hyperactivity-disorder (ADHD) in children: Rating the evidence and proposed guidelines. Applied Psychophysiol ogy and Biofeedback, 45(2), 39-48. https://doi.org/10.1007 /s10484-020-09455-2

Arns, M., Drinkenburg, W., \& Kenemans, J. L. (2012). The effects of QEEG-informed neurofeedback in ADHD: An open-label pilot study. Applied Psychophysiology and Biofeedback, 37(3), 171-180. https://doi.org/10.1007/s10484-012-9191-4

Arns, M., Gunkelman, J., Breteler, M., \& Spronk, D. (2008). EEG phenotypes predict treatment outcome to stimulants in children with ADHD. Journal of Integrative Neuroscience, 7(03), 421438. https://doi.org/10.1142/s0219635208001897

Krepel, N., Egtberts, T., Sack, A. T., Heinrich, H., Ryan, M., \& Arns, M. (2020). A multicenter effectiveness trial of QEEG-informed neurofeedback in ADHD: Replication and treatment prediction. Neurolmage: Clinical, 28, 102399. https://doi.org/10.1016 /j.nicl.2020.102399

Wu, W., Zhang, Y., Jiang, J., Lucas, M. V., Fonzo, G. A., Rolle, C. E., ... Etkin, A. (2020). An electroencephalographic signature predicts antidepressant response in major depression. Nature Biotechnology, 38, 439-447. https://doi.org/10.1038/s41587019-0397-3

Received: December 11, 2020

Accepted: December 11, 2020

Published: December 29, 2020 